\title{
Secondary metabolism and therapeutic efficacy of medicinal plants
}

\author{
A.K. Mohiuddin \\ Assistant Professor, Faculty of Pharmacy, World University of Bangladesh \\ *Corresponding Author: AK. Mohiuddin \\ Email: trymohi@gmail.com
}

\begin{abstract}
Medicinal plants constitute main resource base of almost all the traditional healthcare systems. Most of the herbal drugs produced currently in majority of the developing countries lack proper quality specification and standards. Herbal drugs used in traditional medicine may contain a single herb or combinations of several different herbs believed to have complementary and/or synergistic effects. B oth the raw drugs and the finished herbal products manufactured contain complex mixtures of organic compounds, such as fatty acids, sterols, alkaloids, flavonoids, polyphenols, glycosides, saponins, tannins, terpenes etc. The quality of the finished product is based on the quality of the raw materials. As many as 35\% of the medicinal plants used in Indian systems of medicine are highly cross pollinated which indicate the existence of a wide range of genetic variability in the populations of these medicinal plant species which in turn reflected in the variations in the composition of secondary metabolites. Ecological and edaphic as well as seasonal variations also cause changes in the chemical composition of medicinal plants. These facts have to be considered while developing quality parameters! standards of medicinal plants and their finished products.
\end{abstract}

Keywords: Medicinal plants, Organic compounds, Secondary metabolite, Pollens, Biosynthesis.

\section{Introduction}

While in food plants our main interest is the carbohydrate/sugars, proteins, fats and other vitamins, in medicinal plants we look for therapeutically useful chemicals which are generally termed as secondary metabolites which are not that essential for the normal growth and development of the plants/organisms. Plants synthesize these compounds to protect themselves i.e. to adjust, adapt or defend/offend, from the hostile organisms or diseases or the environment. Secondary metabolites that are useful in medicine are mostly polyphenols, alkaloids, glycosides, terpenes, flavonoids, coumarins, tannins etc. The production of secondary metabolites although controlled by genes but their specific expression is greatly influenced by various factors including biotic and abiotic environments such as climate and edaphic factors or other associated living organisms. During the course of evolution plants have evolved various physical and chemical mechanisms to protect themselves from the vagaries of nature (drought, heat, rain, flood, etc.) and also to defend or offend the predators or to protect from predators and pathogens. The most successful adaptation of plants while developing various physiological mechanisms is through the production of a variety of phytochemicals by which they were able to face both biotic and abiotic stresses and threats. In this process of defense/offence from abiotic stress or the invading diseases causing organisms or the predators (animals, birds, insects and herbivorous animals), the plant synthesize a variety of chemical compounds. Apparently, plants produce many antioxidants for protecting themselves from the oxidative stress. These compounds are in general stored in the leaves or other parts such as, bark, hardwood, fruits, etc., so that the predators or the disease-causing organisms can be either knocked down or paralyzed or even get killed. In many cases, the production of the secondary metabolites in plant also depends on the association of other living organisms, more particularly, the plant or soil microbes. Such differential expressions of therapeutically active principles in plant on account of the above said factors appears to have known and well understood by the ancient scholars, when they gave specific instructions in the procurement of medicinal plants.

\section{Biosynthesis}

The pathways of biosynthesis are responsible for the occurrence of both primary and secondary metabolites illustrated in Fig. 1. Biosynthetic reactions are energy consuming, fueled by the energy released by glycolysis of carbohydrates and through the citric acid cycle. Oxidation of glucose, fatty acids and amino acids results in ATP (adenosine triphosphate) formation, which is a high-energy molecule formed by catabolism of primary compounds. ATP is recycled in fuel anabolic reactions involving intermediate molecules on the pathways. Whereas, catabolism involves oxidation of starting molecules, biosynthesis or anabolism involves reduction reaction. Hence, the need of reducing agent or hydrogen donor, which is usually the NADP (nicotinamide adenine dinucleotide phosphate). These catalysts are known as coenzymes and the most widely occurring is CoA (coenzyme A) made up of ADP (adenosine diphosphate) and pantetheine phosphate. The most common pathways taken for biosynthesis are performed through the pentose for glycosides, polysaccharides; shikimic acid for phenols, tannins, aromatic alkaloids; acetate-malonate for phenols and alkaloids and mevalonic acid for terpenes, steroids and alkaloids. As showed in the Fig. 1, the scheme outlines how metabolites from the process of photosynthesis, glycolysis and Krebs cycle are tapped off from energy-generating process to provide biosynthetic intermediates. By far, the important building blocks employed in the biosynthesis of secondary metabolites are derived from acetyl-CoA (acetyl 
coenzyme A), shikimic acid, mevalonic acid and 1- deoxylulose 5-phosphate. ${ }^{1-7}$

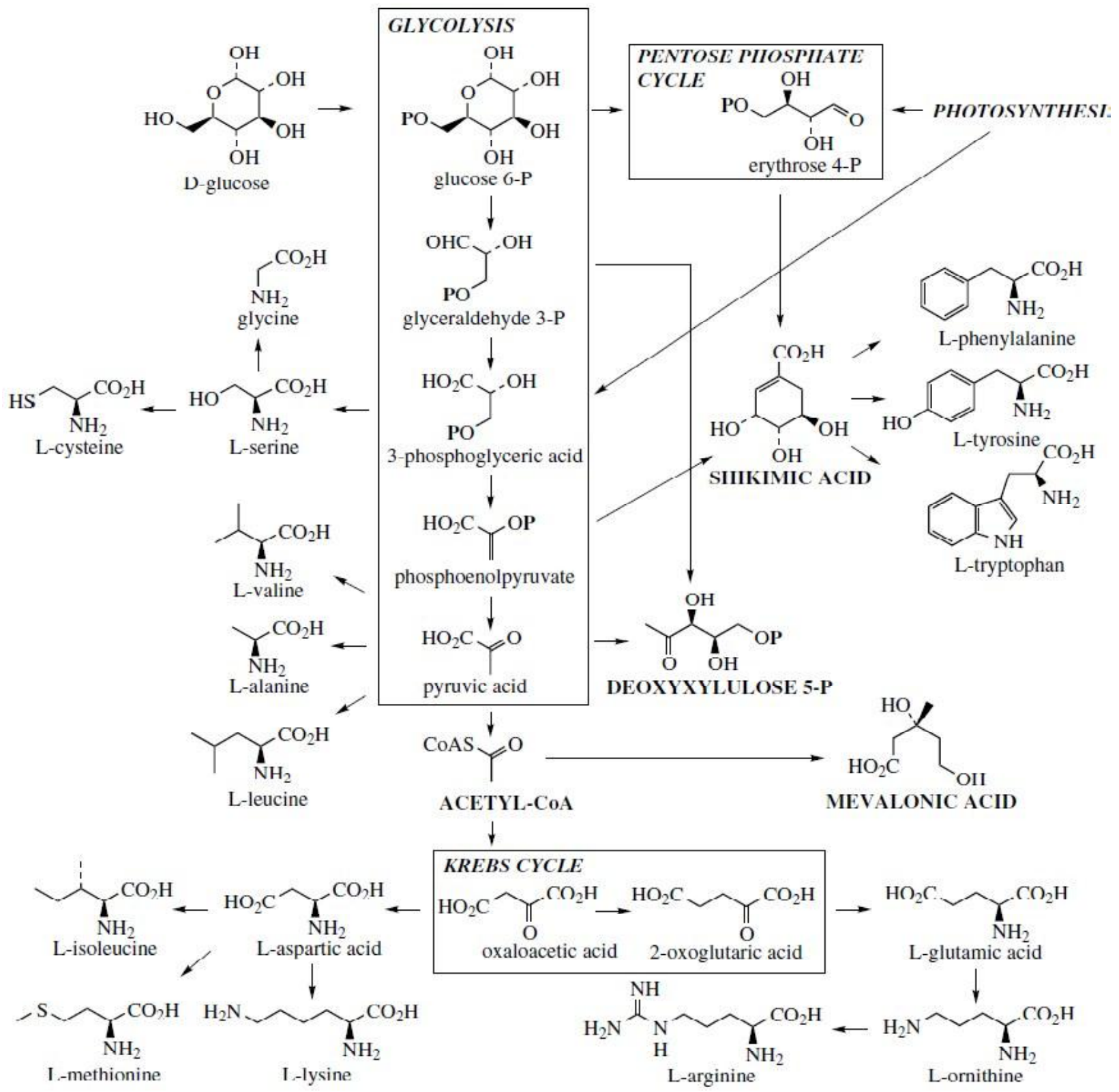

Fig. 1: Biosynthesis scheme of plants secondary metabolites. ${ }^{8}$

\section{Expression of secondary metabolites}

The presence of or absence of certain secondary metabolites in medicinal plants are influenced by a variety of factors, which include climate/season, edaphic conditions or the association of other plants and other living organisms [9]. Another factor that influenced the production of secondary metabolites in plants are the inter relationship between plants and the insect flora. It is now generally accepted that the flora and the insect flora in a tropical ecosystem have been co-evolving and co-adapting. Many of the medicinal plants are cross-pollinated and they need the help of pollinators. In an open area the wind could do the function, but in a canopied forest many of the shrubs and herbs growing under the big trees cannot get wind to pollinate. These plants are thus heavily depending upon the insects or even the birds to pollinate them. To attract the insects or birds the plants develop pleasant aroma (essential oils) and provide honey and pollen as food to these pollinators. Many flowers contain honey or pollen, which are the normal food of many insects and birds ${ }^{[10,11]}$. The insects like bees and butterflies visit flowers after flowers, and take honey or pollen or both. During this process they also carry pollen on their body part, which then help in pollinating while visiting other plants. Many flowers have structurally evolved flower parts to affect such pollinations by insects. These insects also multiply on plants. They lay millions of eggs and the larvae that emerge from these eggs 
then feed on leaves of the plants, sometimes destroying the plants altogether by over feeding. During the course of evolution, the plants began to synthesize certain toxic substance so that a good percentage of the feeding larvae could be killed. ${ }^{12,13,14}$ The insect on the other hand began to develop resistance so that many of the larvae could survive. The plants on the other hand again counteracted. It synthesizing more and more toxic compounds. This was something like the love and hate relationship between plants and the insects, which during the course of millions of years of evolutions have resulted in the synthesis of innumerable chemical compounds, mostly the secondary metabolites in plants as well as in insects. ${ }^{15,16}$ The variability in living organisms is indeed the insurance for survival. The evolutionary origin of cross breeding was indeed a nature's device for reshuffling of genes so that new variants could be produced. Similarly, the abiotic conditions also exerted certain influence in the plants and the plants responded by developing various chemicals. ${ }^{17}$ In extreme drought conditions the desert exerts a kind of stress on the plants and the plants evolve by synthesizing chemicals that would help them to protect from stress induced by the desert conditions. An excellent example for this is the plant Commiphora wightii; an important medicinal plant used extensively as complimentary medicine named 'Guggul'. The medicinal part of the plant is the gum exudates from the stem bark of living plants. This gum is traditionally collected from the desert regions of Rajasthan, Gujarat and even Afghanistan. To everyone's surprise the chemical data of this gum revealed that it does not contain most of the active compounds. A logical explanation may be that this plant growing in a warm humid tropical forest region. It has no desert like conditions and therefore there is no question of any drought induced stress. The same plant when growing in desert has to confront drought induced stress and the plant synthesizes the stress beating chemicals. There are many similar cases that demonstrate that certain specific climatic conditions and edaphic situations are extremely important in the production of therapeutically desirable medicinal compounds. Sandalwood is another classical example. The specific aroma of sandalwood is due to the presence of certain essential oil chemicals, mostly monoterpenes and sesquiterpenes. The production of the specific aroma chemicals is fully expressed only in those sandalwood trees that grow in certain forest regions of Karnataka. The sandalwood growing in other places in India or elsewhere in the world do not have the same kind of aroma with the corresponding chemical constituents. ${ }^{18,19,20}$

\section{Importance of Secondary Metabolites}

Secondary metabolites, which are a characteristic feature of plants, are especially important and can protect plants against a wide variety of microorganisms (viruses, bacteria, fungi) and herbivores (arthropods, vertebrates). As is the situation with all defense systems of plants and animals, a few specialized pathogens have evolved in plants and have overcome the chemical defense barrier. Secondary metabolites, including antibiotics, are produced in nature and serve survival functions for the organisms producing them. Secondary metabolites serve:

1. As competitive weapons used against other bacteria, fungi, amoebae, plants, insects, and large animals;

2. As metal transporting agents;

3. As agents of symbiosis between microbes and plants, nematodes, insects, and higher animals;

4. As sexual hormones; and

5. As differentiation effectors.

Although antibiotics are not obligatory for sporulation, some secondary metabolites (including antibiotics) stimulate spore formation and inhibit or stimulate germination. Formation of secondary metabolites and spores are regulated by similar factors. Thus, the secondary metabolite can:

1. Slow down germination of spores until a less competitive environment and more favorable conditions for growth exist;

2. Protect the dormant or initiated spore from consumption by amoebae; or

3. Cleanse the immediate environment of competing microorganisms during germination ${ }^{[21,22,23,24]}$.

\section{Conclusion}

Secondary metabolites are the useful natural products that are synthesized through secondary metabolism in the plants. The production of some secondary metabolites is linked to the induction of morphological differentiation and it appears that as the cells undergo morphological differentiation and maturation during plant growth. It is observed that in-Vitro production of secondary metabolites is much higher from differentiated tissues when compared to non-differentiated or less-differentiated tissues. There are lots of advantages of these metabolites like there is recovery of the products will be easy and plant cultures are particularly useful in case of plants which are difficult or expensive and selection of cell lines for high yield of secondary metabolites will be easy. Many other examples could be presented with plant metabolic engineering as this research area is developing actively. Metabolic engineering is probably a large step forward but playing on the genes will not solve all the problems that have prevented the development of commercial success in the field of plant secondary metabolites. And Advances in plant cell cultures could provide new means for the cost-effective, commercial production of even rare or exotic plants, their cells, and the chemicals that they will produce. Knowledge of the biosynthetic pathways of desired compounds in plants as well as of cultures is often still rudimentary, and strategies are consequently needed to develop information based on a cellular and molecular level. Because of the complex and incompletely understood nature of plant cells in-vitro cultures, case-by-case studies have been used to explain the problems occurring in the production of secondary metabolites from cultured plant cells. Advance research has succeeded in producing a wide range of valuable secondary phytochemical in unorganized callus or suspension cultures 
till to date; in other cases, production requires more differentiated micro plant or organ cultures.

Purpose of the study: An illustrated review of factors that influence secondary metabolism of plants and their impacts on therapeutic efficacy.

Findings: Traditional plants are used from ancient time for various human well-being, both as life-saving and lifestyle drugs. A careful observation may explore facts behind therapeutic efficacy of these plants.

Materials and methods: A comprehensive literature review, consulting books, technical newsletters, herb magazines, journals, and many other sources. Health professionals like qualified doctors, herbal specialists, folk healers, alternative medicine specialists given their valuable suggestions.

Research limitations: The limitation lies with the unlimited information about traditional medicines. Validity of those are very hard to prove. Only data obtained from books, newsletters, national and international research-based articles are given here along with surrounding facts mostly visible. A few many plant medicine books consulted earlier but article has fewer scope to add from them.

Practical implication: the article is based on plant medicine which is a specialized topic. Students, researchers and professionals may acquire much from this article.

\section{Acknowledgement}

Being a pharmacist in teaching profession is a duty towards future pharmacists as well as other healthcare providers of a nation. And a very good payback was also received from professionals, students and colleagues. Pharmacists of all disciplines that I have conducted was very much helpful in providing books, journals, newsletters and precious time. The greatest help was from my students who paid interest in my topic as class lecture and encouraged to write such article comprising patient communication. Despite a great scarcity of funding this purpose from any authority, the experience was good enough to carry on research.

\section{Conflict of interests: None}

Funding: Funding from individual/Organization: N/A

\section{References}

1. Lehninger Principles of Biochemistry 6th Edition David. L. Nelson and Michael. M. Cox Publisher: W.H. Freeman, 2013.

2. Fundamentals of Biochemistry: Life at the Molecular Level, Donald Voet, Judith G. Voet, Charlotte W. Pratt Publisher: Hoboken, NJ: John Wiley and Sons, 2016.

3. Trease and Evans Pharmacognosy William Charles Evans, Daphne Evans, George Edward Trease Publisher: St. Louis: Elsevier Health Sciences UK, 2014.

4. Pharmacognosy and Pharmacobiotechnology Author: James E Robbers; Marilyn K Speedie; Varro E Tyler Publisher: Baltimore: Williams and Wilkins, (C)1996.

5. Fundamentals of Pharmacognosy and Phytotherapy Author: Michael Heinrich; Joanne Barnes; Simon Gibbons; Elizabeth M Williamson Publisher: Churchill Livingstone, 2012.
6. Medicinal Plants: Ethno-Uses to Biotechnology Era Author: Aly Farag El Sheikha Affiliation: Department of Biology, McMaster University, 1280 Main St. West, Hamilton, ON, L8S 4K1, Canada.

7. Medicinal Plants: Ethno-Uses to Biotechnology Era Author: Aly Farag El Sheikha Affiliation: Department of Biology, McMaster University, 1280 Main St. West, Hamilton, ON, L8S 4K1, Canada.

8. Medicinal Plants: Chemistry and Properties M Daniel Publisher: CRC Press, Apr 19, 2016.

9. Giweli, A. A., Džamić, A. M., Soković, M., Ristić, M., Janaćković, P., and Marin, P. 2013. "The Chemical Composition, Antimicrobial and Antioxidant Activities of the Essential Oil of Salvia fruticosa Growing Wild in Libya." Arch Biolog Sci 1 (65): 321-9.

10. Bruno Leite Sampaio, RuAngelie Edrada-Ebel and Fernando Batista Da Costa Effect of the environment on the secondary metabolic profile of Tithonia diversifolia: a model for environmental metabolomics of plants Sci Rep. 2016; 6: 29265. doi: [10.1038/srep29265]

11. PMCID: PMC4935878 PMID: 27383265

12. Claire Brittain, Claire Kremen, Andrea Garber, AlexandraMaria Klein Pollination and Plant Resources Change the Nutritional Quality of Almonds for Human Health PLoS One. 2014; 9(2): e90082. doi: [10.1371/journal.pone.0090082] PMCID: PMC3937406 PMID: 24587215,

13. Koppert Biological System Why do pollinators visit flowers? URL: https://www.koppert.com/pollination/naturalpollination-bumble-bees/why-do-pollinators-visit-flowers/

14. Ferris Jabr Farming a Toxin to Keep Crops Healthy Scientific American The Sciences September 3, 2013 URL: https://www.scientificamerican.com/article/farming-a-toxin/

15. Forest Health Handbook North Carolina Forest Service 3rd Edition Publisher: Ryan A. Blaedow September 2011.

16. Crop Scouting Manual 2010 Field Crop Integrated Pest Management Program - University of Wisconsin-Extension Cooperative Extension Service.

17. Douglas J. Futuymaa, and Anurag A. Agrawalb Macroevolution and the biological diversity of plants and herbivores Proc Natl Acad Sci U S A. 2009 Oct 27; 106(43): 18054-18061. doi: [10.1073/pnas.0904106106] PMCID: PMC2775342 PMID: 19815508.

18. Marcia O. Mello and Marcio C. Silva-Filho Plant-insect interactions: an evolutionary arms race between two distinct defense mechanisms Braz J Plant Physiol. 14(2):Londrina May/Aug. 2002 http://dx.doi.org/10.1590/S167704202002000200001.

19. Plant Evolutionary Biology Copyright Publisher Name Springer, Dordrecht Information Springer Science+ Business Media B.V. 1988 DOI https://doi.org/10.1007/978-94-0091207-6.

20. Neeraj Jain, Rajani S. Nadgauda Commiphora wightii (Arnott) Bhandari-A Natural Source of Guggulsterone: Facing a High Risk of Extinction in Its Natural Habitat Am J Plant Sci, 20134(6A):57-68. doi: 10.4236/ajps.2013.46A009.

21. Nakuleshwar Dut Jasuja, Jyoti Choudhary, Preeti Sharama, Nidhi Sharma and Suresh C. Joshi A Review on Bioactive Compounds and Medicinal Uses of Commiphora mukul A Review on Bioactive Compounds and Medicinal Uses of Commiphora mukul. J Plant Sci, 7:113-137. DOI: 10.3923/jps.2012.113.137.

22. MEDICINAL PLANTS Utilisation and Conservation 2nd Revised and Enlarged

23. Edition Editor Prof. Pravin Chandra Trivedi Publisher: Aavishkar Publishers, Distributors

24. Jaipur 302003 (Raj) India.

25. Schäfer H, Wink M. Medicinally important secondary metabolites in recombinant microorganisms or plants: progress 
in alkaloid biosynthesis Biotechnol J. 2009;4(12):1684-703. PMID:19946877 DOI: 10.1002/biot.200900229.

26. Michael Wink Chapter 11 Importance of plant secondary metabolites for protection against insects and microbial infections Advances in Phytomedicine 2006;3:251-268 https://doi.org/10.1016/S1572-557X(06)03011-X.

27. Michael Wink Plant breeding: importance of plant secondary metabolites for protection against pathogens and herbivores
Theoretical and Applied Genetics January 1988;75(2):225-

233.

28. Fumihiko Sato Plant Secondary Metabolism DOI: 10.1002/9780470015902.a0001812.pub2.

How to cite this article: Mohiuddin A.K, Secondary metabolism and therapeutic efficacy of medicinal plants, J Pharm Biolog Sci. October-December, 2018;6(4):104-108 